\title{
Implementing Evidence Into Everyday Practice: Midwifery Leaders' Views About What Helps and Hinders Clinical Innovation.
}

\section{Annemarie De Leo ( $\nabla$ a.deleo@ecu.edu.au )}

Edith Cowan University https://orcid.org/0000-0002-0667-5995

\section{Sara Bayes}

Edith Cowan University

Dianne Bloxome

Edith Cowan University

Janice Butt

King Edward Memorial Hospital

Sadie Geraghty

Edith Cowan University

\section{Research}

Keywords: midwifery, Evidence-Based Practice (EBP), evidence-practice gap, Implementation Science, Behavioural theory

Posted Date: July 7th, 2020

DOl: https://doi.org/10.21203/rs.3.rs-37978/v1

License: (c) (i) This work is licensed under a Creative Commons Attribution 4.0 International License. Read Full License 


\section{Abstract}

\section{Background}

Despite the ongoing production of new scientific evidence in the field of maternity care, midwives continue to face challenges when translating latest evidence into evidence-informed care, and report uncertainty in both knowledge and confidence to implement sustained evidence-based change in clinical areas. This study aimed to

explore midwifery leaders' views on what factors help or hinder midwives' efforts to implement evidencebased practices, and test the usability of the Capability, Opportunity, Motivation and Behaviour model and Transtheoretical Domains Framework to analyse the barriers and facilitators of evidence-based change.

\section{Methods}

This qualitative study formed part of a larger Participatory Action Research (PAR) project designed to improve the processes by which midwives implement evidence-based practice change in clinical areas. Data were obtained by a nominal group workshop and individual face-to-face interviews between July September 2019. Thematic analysis was used to interpret the transcribed data, which were then coded and categorised into meaningful themes.

\section{Results}

One overarching core finding emerged from four major categories to fulfil the aim of this study: Fear can stop change and midwives lack the confidence and time to implement new evidence-based practices, however having high level midwives in leadership roles is a huge advantage.

\section{Conclusion}

This study confirms what is known about the hinderances and helpers of translating latest evidence into everyday practice, and provides valuable insight into the usability of behavioural theories and context assessment tools to both diagnose and develop intervention strategies applicable to midwives initiating evidence-based practice change in clinical areas.

\section{Introduction}

The benefits of using evidence-based practices in healthcare have been well reported in the literature (Friesen-Storms, Moser, van der Loo, Beurskens, \& Bours, 2015). Similarly, over the past decade researchers and clinicians have articulated growing interest in mechanisms that improve the movement of best available evidence into everyday practices and policy (Kislov, Waterman, Harvey, \& Boaden, 2014). These mechanisms are largely derived from the field of Implementation Science (IS), which was developed in the 2000's with the ambition to generate knowledge that promotes improved clinical practice through the timely, effective and sustained implementation of evidence-based interventions in a range of 
settings (Tabak, Khoong, Chambers, \& Brownson, 2012). However, despite the development of over 60 implementation theories, models and frameworks, the evidence-practice gap in healthcare remains persistent, as demonstrated in literature that describes the difficulties experienced by clinicians trying to improve practice and processes in clinical areas (Gallen, Kodate, \& Casey, 2019; Heydari, Mazlom, Ranjbar, \& Scurlock-Evans, 2014). Low rates of evidence adoption and inconsistent use of EvidenceBased Practice (EBP) remains a challenge in healthcare after more than two decades of IS research (Pazandeh, Huss, Hirst, House, \& Baghban, 2015).

\section{Background}

Midwifery is a research-informed profession and midwives are expected to apply best available evidence to clinical practice and decision-making. In many countries this is included in mandatory standards for the midwife (NMBA, 2018). Evidence implementation knowledge is, however, not commonly taught in midwifery education and although literature on the topic is continually growing, it does not tend to provide guidance on how to use IS processes to support practice change initiatives (Nilsen, Neher, Ellstrom, \& Gardner, 2017). A key reason for the continued evidence-practice gap in midwifery, like other areas of healthcare, is the limited focus on identifying factors critical to the successful implementation of evidence-based practices (Handley, Gorukanti, \& Cattamanchi, 2016).

One of the fundamental tenants of IS is that closure of evidence-practice gaps requires consideration for mechanisms that are applicable to real-world situations and target both individual and organisational factors influential to the translation of latest evidence into clinical areas (Birken et al., 2017). To achieve this, the factors that both help or hinder the implementation process must be uncovered and addressed, as knowing these will guide clinicians' efforts to optimise clinical innovation outcomes (Vogel et al., 2016).

Although there is an established body of evidence on the barriers and facilitators of EBP in healthcare, there remains limited knowledge of the factors that help or hinder midwives' efforts to implement new evidence-based practices in clinical areas (Azmoude, Aradmehr, \& Dehghani, 2018). Further, little is known about midwives' use of implementation tools to facilitate the process (Bayes, Fenwick, \& Jennings, 2016). The purpose of this study was to address this uncertainty by exploring midwifery leaders' experience of implementing evidence-based practice change.

\section{Aims}

The aims of the study reported in this paper were to explore midwifery leaders' views on what factors helped or hindered midwives' efforts to implement evidence-based practices, and to test the usability of the Capability, Opportunity, Motivation and Behaviour model (COM-B) and Transtheoretical Domains Framework (TDF) to analysis the barriers of evidence-based practice change.

\section{Methods}


This study formed part of a broader Participatory Action Research (PAR) project designed to improve the processes by which midwives implement evidence-based practice change in clinical areas. The study employed a qualitative study design whereby a nominal group workshop (focus group) and individual face-to-face interviews were conducted to gain insight into the views of eight midwifery change-leaders, who all had extensive experience of implementing practice change at both individual and organisational levels. Unlike quantitative research, qualitative inquiry encourages participants to talk freely and often around emotionally laden issues in order to obtain a deeper understanding of how people think and feel about real-world problems. In this respect, qualitative research can be described as conversation with a purpose (Krefting, 1991). Arguably, qualitative studies are able to probe in more depth around a particular issue and thus are more attuned to the verbal and non-verbal cues of the persons involved (Schneider \& Whitehead, 2013). This method of inquiry was beneficial for achieving the aim outlined in this study.

\subsection{Study design}

An exploratory qualitative study was conducted based on one nominal group workshop and five in-depth interviews, all of which were underpinned by the methodological reasoning of Action Research (Kemmis \& McTaggart, 1988). This design enabled the authors to gain an insiders perspective about the midwifery issues uncovered during the data collection process.

\subsection{Ethical considerations}

The Human Research and Ethics Committee at XXXXXX in XXXXXX approved the study. No risks to the participants or the researchers were anticipated and none eventuated.

\subsection{Participants}

The participants were recruited as part of a wider Participatory Action Research (PAR) project designed to improve the processes by which midwives implement evidence-based practices. Eight midwifery experts with experience in leading practice change initiatives were nominated by their managing Directors to participate in the study. These midwifery leaders were sent an electronic invitation to participate, a plain language information sheet and a consent form. All nominated midwives accepted the invitation to participate and consented either to take part in a nominal group workshop or an individual face-to-face interview depending on their work commitments.

\subsection{Data collection}

The Nominal Group Technique (NGT) was used in the workshop to achieve general agreement and convergence of opinion relating to several discussion points derived from the aim outlined in this study. Commonly referred to as a consensus technique for establishing priority information, the NGT was used to direct problem solving, generate ideas and prioritise action planning (Harvey \& Holmes, 2012). A key strength of conducting a nominal group workshop was the balanced contribution of all participants and the semi-structured format of the group discussion. The NGT also aligns with the constructs of qualitative research in that it has proven more effective in obtaining participant responses in greater 
depth and breadth compared with traditional focus group discussions (Langford, Schoenfeld, \& Izzo, 2002). The nominal group workshop was co-facilitated by (BLINDED FOR PEER REVIEW) over three hours, wherein discussions were audio-recorded with consent and additional fieldnotes were documented throughout the session. All participants were ascribed pseudonyms.

Due to work commitments, three participants opted to participate in audio-recorded individual face-toface interviews that focused on the same discussion points explored at the nominal group workshop. In person interviews were preferred over telephone interviews because observation and recording of both verbal and non-verbal communication was possible, and the interviewer could improvise to elicit clearer and more elaborate responses from participants when needed (McIntosh \& Morse, 2015).

\subsection{Data analysis}

The audio-recordings and field notes from the nominal group workshop and face-to-face interviews were transcribed and analysed ('coded') as soon as possible afterwards. This was through a process of reflexive thematic analysis as described in Braun and Clarke's approach (Braun \& Clarke, 2006). This qualitative method involved initial familiarisation with the data, coding of key statements and then grouping emergent themes and patterns within the data into meaningful categories. These categories were presented on a storyboard to the research team where additional refinement of them occurred. The final iteration of the findings categories and their constituent data were unanimously agreed on by all members of the research team. The analysed data were then mapped to the 'Capability, Opportunity, Motivation and Behaviour' model or 'COM-B' (also recognised as the 'Behaviour Change Wheel' or 'BCW') (Michie, van Stralen, \& West, 2011) and the 'Transtheoretical Domains Framework' or 'TDF' (Cane, O'Connor, \& Michie, 2012).

This method of data analysis resonates with existing literature reporting on the barriers and facilitators of change in healthcare whereby influential factors, consistently reported as communication processes, workplace culture, practitioner skills, individual attitudes and organisational 'readiness' for change, have been grouped into three main domains: staff, systems and intervention (Geerligs, Rankin, Shepherd, \& Butow, 2018). Collectively these domains represent the multi-dimensional factors that influence the success or failure of evidence-based change implementation efforts.

Although IS is a relatively new field in healthcare, clinicians seeking guidance for implementing evidencebased innovations can select from a range of IS processes and strategies, designed to support the uptake and longevity of EBP in healthcare (Moullin, Sabater-Hernandez, Fernandez-Llimos, \& Benrimoj, 2015). More recently the implementation of behaviour change interventions (such as implementing new evidence-based practices) are recognised to be more effective when based on theory, compared with those that lack a philosophical approach (Glanz \& Bishop, 2010; Hanbury \& Wood, 2018). One such behavioural theory, the 'Capability, Opportunity, Motivation and Behaviour' model ('COM-B'), also recognised as the 'Behaviour Change Wheel' ('BCW'), identifies systems that energise and motivate behaviours at both an individual and organisational level (Michie et al., 2011). The key premise of the COM-B is in understanding that Capabilities (an individual's capacity to engage in behaviour 
modifications), Opportunity (factors in the environment that influence individual behaviours) and Motivation (the willingness to change) generate behaviours which, if identified, can be leveraged to influence Behaviour change interventions (Handley et al., 2016). In essence, the COM-B model highlights that in order to adopt new behaviours or practices an individual needs to be capable of change, have the opportunity to change and have the motivation to engage in new behaviours or practices. However, given the abundance of behavioural theories currently available to guide innovations that involve behavioural change (Davis, Campbell, Hildon, Hobbs, \& Michie, 2015), clinicians face a dilemma when trying to select the most appropriate theory to optimise implementation outcomes. Context assessment frameworks derived from IS research may provide a solution to this dilemma. Implementation Science frameworks have the capacity to embed theoretical perspectives into the implementation process, effectively mapping behavioural theory to a contextual framework tool.

One example is the Transtheoretical Domains Framework (TDF), which builds on the systems identified in the COM-B to further break down what underpins individual-level barriers and facilitators of change, and enhances the development of tailored intervention strategies by enabling the practical use of theory in clinical environments (Cane et al., 2012). Comprising 14 (originally 12) theoretical domains, the TDF is a well-validated, comprehensive framework that can be used to assess the barriers and enablers of behaviour change as well as 'diagnose' the social and contextual factors that may influence the change implementation process (Michie et al., 2005). The domains cover three broad factors: individual-level variables, social/cultural variables, and system-level variables, all which can be mapped to the COM-B systems (Table 2). The TDF is particularly applicable to those wanting to adopt a more systematic approach to problem-solving and refine the design of intervention strategies specific to real-world problems (Fuller et al., 2014).

While using a single theory or framework may be appropriate to solve implementation issues in some healthcare contexts, midwifery is a highly specialised profession where midwives work in varied clinical areas and may be subjected to numerous individual, organisational and contextual factors that influence their efforts to implement evidence-based practice change. The COM-B and TDF are two strategies that, when used in combination, may be useful tools for midwives wanting to optimise the efficiency and outcomes of implementing EBP in clinical areas.

\subsection{Trustworthiness}

The research team sought to assure the study's methodological trustworthiness in three ways. Firstly, a detailed audit trail was recorded whereby all steps of the data analysis process were tabulated and clearly reported to capture each stage of thematic analysis. Second, major analytical decisions were discussed between the research team on three occasions to progressively refine the emergent categories. Finally, a member checking exercise was conducted wherein the final categories derived from the data used to explore the phenomenon of interest were presented to academic midwifery colleagues to ensure that the analysis and emergent findings were a clear and accurate representation of the findings reported in this study. 


\section{Results}

A collective of eight midwifery leaders who all had expert experience of either overseeing or implementing a practice change in their workplace took part in this study. Participants shared experiences from both metropolitan and rural clinical sites, which represented all public health sectors of the region. Examples of practice change initiatives ranged from implementing sterile water injections as a method for pain relief during labour, implementing midwifery-led models of care, introducing water birth facilities, overseeing the integration of 'peanut balls' (labour equipment) into birth suites and introducing bedside clinical handover. One overarching core finding, expressed as an interpretive statement, emerged to characterise participants' change leadership experiences and views. Four major categories underpin this finding, which were collapsed from 72 interpretive statements. Three of these major categories represent the factors that hinder midwives' efforts to implement practice change, the other comprises factors that facilitated midwives efforts to introduce and sustain EBP. These are presented in Table 1.

(Please insert table one)

The core finding is presented below, followed by the four major categories and their supporting findings.

5.1 Core finding: "Fear can stop change, and midwives lack the confidence and time to implement new evidence-based practices, however having high level midwives in leadership roles is a huge advantage."

What emerged from the data collected for this study was that fear at an executive level and by the service providers themselves hindered midwives' efforts to initiate and implement sustained practice change. This was influenced by midwives not having the confidence or skills to successfully implement evidencebased practices during worktime and was made more difficult by medical personnel who, to some extent, challenged the implementation efforts of all midwifery leaders. These hindering factors are discussed in detail in major categories one, two and three. Although hindering factors were explored extensively by the expert midwifery participants, a number of change facilitators were also identified and contribute to the core finding of this study. Executive buy-in and midwifery leader 'champions of change' were considered to be leverages for midwives wanting to initiate evidence-based practice change. This aspect of the findings is reported below in major category four.

5.2 Major category 1: The stumbling block is medical opposition and workplace culture; they are two of the biggest challenges for midwives.

The most significant challenges reported by all midwifery leaders relate to the resistance midwives experienced when trying to introduce new practice or policy changes to clinical areas. This came largely from medical practitioners, but also included management, administration and practicing midwives who obstructed midwifery leaders' efforts to implement new evidence-based practices. MW3 shared the difficulties she experienced when trying to implement waterbirth facilities in her workplace; 'primarily because of...medical opposition, the obstetrician's threw tantrums...literally stormed out of rooms and threatened to withdraw their services... or not attend waterbirths.'This was, she said, made more 
challenging by several midwives labelled "resisters" saying 'all the things the executives wanted to here.... but behind closed doors saying we can't do it now, let's start try it [waterbirth] next year.'MW4 experienced similar challenges when trying to introduce Midwifery Group Practice (MGP) into her organisation. She recalled 'we didn't think it was going to be an issue, well... the medical directors refused to participate... they resisted and they weren't going to let it go.'Reflecting on the struggles she encountered, she questioned 'why do we [midwives] need medical approval for something that is essentially midwifery-led and entirely within our scope of practice?', and stated that in the end 'medical directors were bypassed'to get MGP 'across the line'.

The source of the resistance to practice change the participants encountered was explored by MW7, who offered that 'midwives think it's too much hassle [implementing practice change] and too much work when they're in the middle of a busy shift.'She later continued 'it's difficult to motivate them [midwives] when there's so much change that occurs.' MW1 agreed and added 'our midwives don't necessarily want it [practice change].' These discussions led to participants identifying various contextual factors that influenced change efforts, with work place culture identified as "one of our biggest problems "(MW3). This was exemplified by MW6, who shared her experience of trying to introduce sterile water injections as an option for pain relief in her organisation's birth suites. She experienced 'rumour mongering'from 'people working in the service who did not trust the evidence... it was a cultural thing.'This was made more challenging by staff saying, 'I want to do it, but can't do it now....' and later disclosed "I've received hate mail from people thinking what I wanted to bring in was unsafe... there was so much distrust for a practice that is essentially evidence-based." MW1 had a similar experience and recalled a midwife colleague expressing her anger at a practice change, which included accusing MW1 thus "You're making us jump through hoops" and saying "I won't do it."

MW7 shared her experience of trying to introduce bedside clinical handover in her workplace, stating midwives "seemed keen, but there was an unspoken resistance... and if I wasn't physically present at handover time... it just didn't happen." MW2 agreed and recollected the personal effort required to introduce 'peanut balls' into her birth suites: "we had all the evidence to support this equipment, paid for our midwives to attend... workshops... and demonstrated the peanut balls increased our vaginal birth rates significantly," but expressed frustration at the resistance she experienced from staff: "I still see the peanut balls put in the cupboard three months on and question is change actually happening here?... I have to be onto it, physically checking the rooms to make sure the balls are being used, and this equipment is evidence-based."

There were also practical reasons reported by staff who challenged participants' efforts to implement new evidence-based practices. For example, MW5 stated "[the resistance] wasn't related to what midwifery was about... midwives were concerned by how it would affect them working in practice..." she recalled her midwifery colleagues saying "I would love to do it [MGP], but I only work 0.5 " and "it's a good idea, but I just don't have the time." This resonated with MW6, who shared her thoughts on why she experienced difficulty getting compliance with new practice and policy within her workplace "it's just that [the midwives are] too busy delivering direct care... they work hard and there are too many barriers." 
The overarching essence of this major category is reflected in a comment by MW5, who suggested that the degree of success change leaders have when trying to implement new evidence-based practices relates to having "good evidence...removing the emotions and just playing it out." This can be challenging for midwives as fear and personal attributes were also reportedly put forward by participants to be hindrances of practice change. These factors are explored in more depth in the next major category.

\subsection{Major category 2: Fear can stop change: it's personal for midwives.}

In addition to the inter-professional and collegial opposition experienced by all midwifery leaders, fear was identified as a significant barrier to implementing new practice innovations. For many midwives, this was derived from distrust and not understanding how introducing a practice change worked operationally. MW3 described this when she shared her story of trying to implement water birth facilities; "part was not understanding how it [water birth] would affect what midwives had to do and how it [waterbirth] would affect midwives working rosters.'She went on further to say that "people didn't really have an understanding of it [water birth] and that brought about a fear, and when there is fear that stops change." MW1 had similar experiences trying to implement water birth in her labour unit; she recalled staff saying "I don't trust the evidence" and asking "does this mean more paperwork?", which caused trepidation "as staff struggled to accept a practice that is happening in similar environments around the world."

MW4 told a similar story of the challenges she experienced when trying to introduce MGP within her organisation. She reported midwives questioning "how is this going to affect my income and time away from work? [And] when it came to actually signing up for MGP, fear came in...and some of that is personality, which was what I really believe held up the process." MW7 concurred and offered, "midwives want to know why, even when they've been shown the evidence... and they want to know how it affects them on the floor."

What resonated between the stories of all participants was that fear hinders change, and as a consequence change takes time. MW2 suggested "what I've learned is that operationally, change takes about 12 months and is a continual cycle of thinking, reviewing and revisiting the initiative to ensure its sustainability." MW3 agreed, stating her implementation efforts took "...8 months and even 10 years on, it's still hard work and the criticisms keep coming." All midwifery leaders agreed with MW8, who suggested "change is sometimes not a good fit for the organisation...it's personal for staff." This resonated with MW7 who said "some of it's personality...and the behaviours of individuals can affect the success of change initiatives." She went on to state "it also depends where the change is coming from...is it being driven by us [midwives]... or is it being driven by the organization?"

Two midwifery leaders reported they had better success with introducing evidence-based practices when the change initiative was midwife-led and midwives were directly involved in the process. MW7 shared her experience of introducing a midwifery-led antenatal clinic, stating "we sat down with the staff and 
identified the problems they were having... and asked them [midwives] what they wanted to do to improve the situation." On reflection, she said "I think because it was a midwife-led initiative we had instant buyin... and that made a huge difference to the outcome of the project." MW5 concurred, sharing her experience of introducing bedside clinical handover: "involving the staff seemed to embed the change more easily" because "when there's a perceived threat to midwives family time or income... there's distrust, and fears comes in." MW4 disclosed it was her midwifery colleagues that caused the most resistance and tried to sabotage her efforts to introduce MGP services. She stated "[midwives] got in under me... cut the reeds down while I was doing it [implementing change]...you know the culture of women." MW7 suggested to improve the adoption of evidence-based practices "midwives need praise and reassurance to let them know their efforts are being noticed."

The personal effort required to introduce new practice initiatives was reported by all midwifery leaders to be another factor that contributes to the success of change implementation efforts. These data are presented below in major category 3 .

5.4 Major category 3: Midwives are tired of fighting the battle for EBP; they need knowledge and the confidence to bring about practice change

All participants described varying degrees of hardship when trying to implement practice change, which stemmed not only from the resistance exhibited by staff and management, but also from midwives lacking the confidence and knowledge to efficiently implement evidence-based practices. MW3 told a story of talking with midwives about EBP, and confirmed that "there are still a lot of midwives who are unsure of how to read the evidence... they're not confident with the interpretation despite doing research units at uni." She went on further to question "how do we translate something we can't interpret?" and suggested "we need our midwives to feel confident that they have the ability... and the evidence to defend their practices... and believe in their knowledge." MW7 shared similar experiences with trying to implement practice change and observed "our midwives are fatigued, they work hard and are tired of fighting the battles that change brings." MW6 agreed that "the average midwife doesn't have the time or energy to implement practice change" and went on to articulate "midwives and managers are extremely busy, we need time allocated during work hours to implement new practices..." This resonated with MW4 who also noted that "midwives are time poor and there are less resources and more activity..." She went on to suggest that "changing practice is just not a priority for midwives working day-to-day."

The continual publication of new evidence based practices was considered by MW6 to be challenging for midwives, who acknowledged "in this massive change environment we want to fix everything and do it quickly, but there's so much to fix and midwives are too busy... we don't do change well and there's not enough resources to support us to do it properly." MW3 concurred, and proposed that

...change is something we do badly in everyday practice.... we're very reactionary and don't go back to evaluate... we might change a policy quickly, tell them [midwives] to get on with it and move onto the next job...sometimes that's not easy. 
MW3 suggested the only way to optimise the uptake of change initiatives was to "link change with good evidence and make initiatives not labour intensive." MW7 added "the success of change efforts depends on the resources you've got and the people available to embed initiatives into workplace environments." In addition to all midwifery leaders speaking at length about the hindering factors that impinge on midwives' efforts to successfully implement new evidence-based practices, five out of eight participants also reported leveraging factors that facilitated the implementation process. These are discussed in major category four.

5.5 Major category 4: It all comes down to being the 'squeaky wheel' and having stakeholder buy-in and high level midwives in leadership roles is a huge advantage.

Although participants reported largely on the challenges they experienced with implementing practice change there was also talk of leveraging factors, and how these factors could be used to support midwives efforts to embed latest evidence into everyday practice. MW4 reflected on her efforts to implement MGP, acknowledging that, "to get to the point where we actually introduced change... it was about being the squeaky wheel and getting buy-in from the people who could actually implement the change." Having stakeholder buy-in was also identified as a helping factor by MW1, who stated "having high level midwives in leadership roles... and buy-in at all levels" contributed to the successful implementation water birth in her place of work. MW6 asserted that "getting the right stakeholders on board from the start" is a huge advantage as she recalled the effort required to introduce an evidencebased policy for delayed cord clamping, suggesting "change initiatives have to be endorsed from the top."

Change-leaders' and 'champions of change' were terms used interchangeably by MW8 to describe the value of "having somebody at the top level, a driver of change." MW5 advocated for "an expert in what you are trying to implement" involved in change initiatives, and suggested "you need someone who's had a positive experience so you don't get all that negative stuff that people don't want to hear when they're trying to bring about change." MW7, who talked about the challenges of trying to introduce clinical bedside handover, proposed that "what assisted the change ... was having somebody to keep on driving the project... to lead the initiative and not let it go backwards... because things do with time.

Finally, what resonated between all participants stories was that to successfully embed evidence-based practices, persistence and strong midwifery leadership is required. MW5 surmised "you just chip away at change... even when you're told there's no money or buy-in, change comes with time and focused direction."

\section{Comparison of the findings to the combined COM-B and TDF domains}

As stated earlier, the findings of this study were mapped to the combined COM-B and TDF for further contextual exploration (as exemplified in Table 2). This exercise provided an indication of how effective combining a behavioural theory with a context assessment tool is to assess the helping and hindering factors that might impact evidence-based practice initiatives. It also yielded insight into appropriate intervention strategies that may support midwives' efforts to introduce evidence-based practice in clinical 
areas. What emerged from this mapping exercise is that the change helping or hindering factors that influenced participants' efforts to implement evidence-based practice were related to twelve of the fourteen domains within the TDF.

(please insert table 2)

\section{Capability}

Three of the TDF domains were described by participants in their experience of implementing evidencebased practices within the Capability system of the COMB-B. Namely, these factors were mapped to Knowledge, Skills and Behaviour Regulation within the TDF (domains 1, 2 and 14). Participants described a lack of knowledge amongst midwives with regard to implementation processes. Specifically, participants described most midwives to have limited skills in sourcing, interpreting and translating best available evidence into everyday care. Concerning Behaviour Regulation (domain 14), all midwifery leaders acknowledged the challenges midwives experience introducing change initiatives in addition to their daily workload, and the general consensus was that "change takes time and you need a visible presence... you've got to drive it [change initiatives] and that's sometimes not easy" (MW7). Further, two midwifery leaders acknowledged the importance of ongoing audit and evaluation to ensure change initiatives were sustained.

\section{Opportunity}

Two of the TDF domains (Environmental Context and Resources and Social Influences) were evident in the transcribed data that were subsequently mapped to the COM-B Opportunity component. Participants talked of local and organisational hindrances (TDF domain 11) within their work environments that hampered midwives' efforts to introduce new evidence-based practices. For example, MW4 identified multiple contextual and social factors that hampered her efforts to introduce Midwifery Group Practice (MGP) during all stages of the project. She commented that the level of success she had with introducing MGP was dependent on "the resources we had at the time... and the people available to actually embed the change."Social Influences were explored by MW5 who recalled conversations with a midwife who said: "that sounds like a great idea, and in a perfect world if I didn't need sleep, have my family and need to pay the bills I would [trial MGP]... let's wait till next year" (MW5). The resistance experienced by all midwifery leaders not only delayed the opportunity to introduce new practices but also lengthened the time it took to embed practice change in clinical areas. This resulted in varied levels of success and also exacerbated the personal stress and fatigue of all midwifery leaders' implementation endeavours.

\section{Motivation}

When mapped to the COM-B, the TDF domains identified in this system included: Beliefs about capabilities (4), Beliefs about consequences (6), Social/professional role and identity (3), Emotion (13), Optimism (5), and Reinforcement (7). What resonated in the transcribed data with these was that midwives lacked the time and confidence to implement new practices, which led to aversion by some 
midwives who were faced with adopting new evidence-based practices. This reflected the beliefs of other midwives, who questioned their capability to initiate practice change and the consequences of adopting new behaviours. MW7 recalled a conversation with one of her midwives, who questioned "why are we changing things again?... we're busy enough already... just don't have the time now" (MW7). Practicing midwives were also reported by participants to be driven by automatic (emotional) responses to change, which often related to their perceived views of how of practice changes would affect their workload and personal time. This was evident in the experiences shared by MW3, who reported conversations with midwives during the implementation of water birth at her place of work. One midwife was quoted to say "I didn't say I don't believe it (the evidence), I just want to know how it's going to affect my family time and income?"Within the constructs of domain 13; stress, fatigue and anxiety were described by the participants in relation to their own evidenced-base change efforts and in their accounts of how practicing midwives who demonstrated mixed feelings of optimism/pessimism towards new evidencebased practices in clinical areas. A core finding of this study is that fear stops change, and the TDF proved valuable in deconstructing this further to underpin the reasoning behind many midwives resistance around introducing practice change and implementing EBP.

Midwives incentives to change were explored under Reinforcement (TDF domain 7). MW6 suggested "there's not enough pre-education to motivate midwives to change... and there are so many changes and innovations... it's difficult to motivate them [midwives] when there is so much change that occurs." Comparatively, MW8 highlighted that when midwives' efforts to adopted new practices were acknowledged, change initiatives seemed to embed more easily into everyday practice. No participants reported the use of other reinforcement techniques as articulated within the constructs of domain 7 . For example, references to the use of rewards, punishment, consequents or sanctions and contingencies were not voiced by any of the midwifery leaders.

Feelings of Optimism (TDF domain 5) resonated in the stories shared by the majority of midwifery leaders, as exemplified by MW8, who said "I think they've (midwives) done amazing [sic] with embracing change... we can't lose sight of that."The constructs within this domain also reflect the Social Professional Role and Identity (TDF domain 12) and professional responsibility of midwives to lead change initiatives in maternity care settings. MW3 reflected on these issues in her experience of implementing waterbirth: "when we lead initiatives we get things done...and we don't do things individually, you need buy-in at all levels... and we have to be united...all in or all out." Additionally, MW6 identified that embedding practice change can also be influenced by other healthcare organisation's policies and practice. For example, when she tried to introduce a policy for sterile water injection for pain relief during labour MW6 argued with management: "this is what other hospitals are doing nationally and internationally, why are we so behind?... and that's how we got it across the line."

\section{Discussion}

The eight participant midwifery leaders' practice change experiences heard in this study clearly illustrate the hindering and helping factors that typically contributed to the success or otherwise of implementing 
evidence-based practice change in various maternity practice settings. The data were represented in four major categories that broadly characterise the individual, workplace and system level factors that either stalled or were used as leverages to embed EBP. Issues of fear, fatigue, medical opposition, lack of time and limited knowledge of implementation processes were reported as hindering factors.

Comparatively, having strong leadership and a solid evidence-base were highlighted to facilitate the implementation efforts of midwifery change-leaders in clinical areas. Only three publications relating to implementation challenges in midwifery could be sourced against which to compare what we discovered, and the findings of those studies broadly resonate with the helping and hindering factors identified in this study (Bayes, Juggins, Whitehead, \& De Leo, 2019; Nithianandan et al., 2016; Tracy, Hartz, Nicholl, McCann, \& Latta, 2005). To date, there has been only one publication exploring the use of IS tools in midwifery contexts. Bayes et al (Bayes et al., 2016) tested the Consolidated Framework for Implementation Research (CFIR), a seminal context assessment tool, to determine the usability of IS tools in midwifery practice contexts to assess organisational readiness for practice change. Bayes and team (Bayes et al., 2016) proposed that the CFIR is beneficial for conducting broad contextual assessments, but that the language used in it renders the tool inappropriate for midwifery contexts in its original form. Bayes and colleagues recommended the need for increased knowledge and use of IS processes in midwifery, and further research into implementation mechanisms developed specifically for the needs of practicing midwives wanting to implement evidence-based practices.

There has seemingly been no other work describing the use of either the COM-B or TDF in midwifery settings, however there is a small number of published studies (three) that broadly report on the use of the TDF and COM-B in other clinical environments. In their study of healthcare workers' perceptions of why patients were or were not assessed for rehabilitation after undergoing a stroke, Lynch et al (2017) employed the TDF to explore the factors that contributed to assessment and referral practices by healthcare workers in acute stroke units. Key factors influencing practice were identified and mapped to the TDF domains (this included individual and organisational influences and practitioner skill and knowledge deficits), which were then assessed by participants to determine the suitability of employing the TDF to improve rehabilitation and referral practices for patients. Key findings highlighted the potential for using the TDF to identify factors that influence clinician practices. Additionally, Lynch and associates articulated the value of using the TDF to enhance clinical outcomes of patients. Recommendations for further research focusing on strategies that integrate EBP into routine systems was also advocated to optimise the use of latest evidence in practice environments. Although this study provided relevant information on the usability of the TDF, the authors did not make reference to the use of behavioural theories to validate their findings.

Flemming et al (Fleming, Bradley, Cullinan, \& Byrne, 2014), in their study investigating healthcare professionals' views on antibiotic stewardship (over-prescribing) in long-term care facilities, combined the TDF with the COM-B and behaviour change technique taxonomy to map the influential factors that contributed to the over-prescribing of antibiotics by doctors working in clinical areas. Participants (comprising doctors, pharmacists and nursing staff) identified several barriers that influenced their 
prescribing practices: lack of knowledge, skills, inconsistency in individual practices, miscommunication and unsupportive workplace culture, all of which resonate with the findings of our study. Combining the COM-B and TDF apparently proved useful in this study and the authors reported that the tools effectively "identified the challenges... along with many broad issues at play...on which to model future antimicrobial stewardship interventions' ( $p$. 9). Further, these authors recommended future studies exploring behavioural change to include both the COM-B and TDF for more detailed analysis of the barriers and facilitators of change.

In the field of dentistry, Asimakopoulou and Newton (Asimakopoulou \& Newton, 2015) explored the use of conventional Behavioural Change Theories (BCT) to optimise the practice of positive behaviours by consumers towards oral hygiene. The authors supported the use of the COM-B in combination with the TDF to identify individual determinants that either enabled or challenged positive behaviours towards oral health, and considered the domains of the TDF useful for developing strategies attuned to the barriers and facilitators of change initiatives. Asimakopoulou and Newton also highlighted the absence of clear guidance for both researchers and clinicians with regard to selecting suitable theories and models; they advised against a 'one-size-fits-all approach,' arguing that implementing interventions that require the adoption of new behaviours warrants consideration for the use of a range of IS tools to accommodate the complexities of interventions involving behaviour modifications. Recommendations for further research into what combination of theories, frameworks and other tools may produce the best outcomes for implementation efforts was highlighted in this study as a priority for future research.

It emerged that none of the participants had considered or used IS methods to support their implementation efforts. It is plausible this reflects the near absence of midwifery research relating to IS processes and offers an explanation for the persistent evidence-practice gap in midwifery practice settings. The core finding to emerge from this study clearly illustrate that fear, lack of confidence and knowledge about implementation processes, and time constraints obstruct midwives' capacity to implement new practices. When applied to the constructs of the TDF these findings were further deconstructed to explore in more detail the various individual, organisational, social and contextual factors that are influential to the implementation and longevity of practice change initiatives. These findings resonate with research reporting on nurses' experiences of implementing EBP (Khammarnia, Haj Mohammadi, Amani, Rezaeian, \& Setoodehzadeh, 2015), where lack of time, staff shortages and a heavy workload were considered the most common barriers to implementing EBP. Similarly, work by Hogan et al (Hogan, Barry, Burke, \& Joyce, 2011), who reported on healthcare professionals' experiences of implementing integrated care pathways, suggest clinicians experience, stakeholder buy-in and communication were also common factors identified as influential to implementing change, which together support the findings of this study.

Finally, only two of the TDF domains were not identified in the findings presented in this study: Intentions and Goals (TDF domains 8 and 9), which may offer some insight into why participants experienced the challenges they reported and provide direction for future implementation processes in midwifery. Although all participants set broad goals to implement an evidence-based practice change, none 
specifically spoke of the methods they used to plan, implement, evaluate and sustain their clinical innovations. This does not imply that these steps were not considered by participants, rather it highlights the need for change-leader midwives to consider goal setting and action planning (also termed 'intervention mapping') in their efforts to embed new evidence-based practices. Although ongoing audit and evaluation were reported by two of our participants, none articulated how they intended to address behaviour change or recognised the value of incorporating IS processes in their implementation efforts.

Research endeavours to identify, develop and refine implementation processes have promised a way to bridge the evidence-practice gap in healthcare and improve the quality of services provided by clinicians working in clinical environments (B. Powell et al., 2012). Relevant to this study, developing effective implementation strategies specific to the needs of midwives is recognised as a priority consideration for midwifery researchers to both support change-leader midwives and improve implementation processes for timely evidence-based change. The Expert Recommendations for Implementing Change (ERIC) project developed a compilation of 68 implementation strategies that provide a foundation for constructing intervention strategies (for example: Education, Training and Environmental restructuring) that are multidimensional and useful for targeting change innovations at both individual and organisational levels (B. J. Powell et al., 2015). Although not context specific, the ERIC implementation strategy compilation may be of use to midwives wanting to target intervention strategies specific to the implementation hindrances and helpers explored in this study.

\subsection{Limitations}

Although the sample provided sufficient data to generate significant findings in this study, the authors acknowledge two limitations. Firstly, the participants represented a relatively small portion of experienced midwifery leaders and may have benefited from including practicing midwives currently working within the service. Secondly, while participants were employed in all public sectors of Western Australian maternity services it is possible that data saturation may not have been reached as a result of the small sample size. Subsequently, it is possible the findings of this study may not reflect the wider implementation issues midwives experience in other midwifery practice contexts.

\section{Conclusions}

The findings of our study identify a disconnection between high level evidence, current midwifery practice and mechanisms known to promote the implementation efforts of midwives seeking to initiate evidencebased practice change. This prompts several recommendations by the authors. First, maternity service providers are advised to scale up their knowledge and confidence to introduce evidence-based practices, because it is the key to moving midwifery forward. Second, organisational commitment to the implementation of evidence-based practice and the introduction of implementation strategies that support midwives' efforts is strongly encouraged. This includes both interdisciplinary and organisational buy-in at both local and organisational levels. Third, the value of strong leadership and 'change champions' cannot be understated. Introducing senior leadership or champions of change, as part of a 
larger 'implementation team' within an organisation may improve the co-ordination and outcomes of change initiatives. Finally, providing midwives with the resources and time to implement change within their practice environments is central to the success of practice change initiatives. Midwives require builtin time to source, interpret and translate latest evidence into EBP, which should be incorporated into their daily workload.

This study is significant in that it confirms what is known about the challenges and successes of translating latest evidence into EBP. We also provide valuable insight into the use of behavioural theories and context assessment tools to both diagnose and develop intervention strategies targeted to the needs of change leader midwives working in clinical areas. Additionally, we tested the value of combining the COM-B with the TDF to diagnose the helpers and hindrances of implementing evidence-based practice change in midwifery for the first time and found it to be extremely helpful. This process enabled an assessment of the effectiveness of these tools when combined and establishes the starting point for developing intervention strategies specific to midwifery practice contexts and the midwives working in these areas. It is anticipated that the findings of this study will lead to mechanisms that support midwives' efforts to translate latest evidence into evidence-based practices. Midwives are key stakeholders in this venture, thus should be consulted and included in future research designed to improve mechanisms that support their implementation efforts and address the evidence-practice gap in midwifery.

\section{Abbreviations}

Implementation Science (IS), Evidence-Based Practice (EBP), 'Capability, Opportunity, Motivation and Behaviour' model (COM-B), Transtheoretical Domains Framework (TDF)

\section{Declarations}

\section{Ethical approval and consent to participate.}

The Human Research and Ethics Committee at XXXXXX in XXXXXX approved the study. No risks to the participants or the researchers were anticipated and none eventuated.

\section{Consent for publication}

This manuscript contains no individual data or personalised information of any persons involved in this study.

\section{Availability of data and materials}

The data sets during and/or analysed during the current study are available from the corresponding author on reasonable request.

\section{Competing interests}


The authors declare there are no financial or non-financial competing interests for this study.

\section{Funding}

No funding was sought for this study.

\section{Authors contributions}

1) A. De Leo: lead researcher and interviewer. Responsible for data collection and analysis, editing, researching and overseeing the research project.

2) S. Bayes: Responsible for data analysis, editing and formatting manuscript.

3) D. Bloxome: Responsible for data analysis and editing and formatting manuscript.

4) J. Butt: Data analysis and editing.

5) S. Geraghty: Responsible for editing.

\section{Acknowledgements}

Thank you to all participants who consented to be a part of this study.

\section{Author's information}

Nil disclosed.

\section{Footnotes}

Nil.

\section{References}

1. Asimakopoulou, K., \& Newton, J. T. (2015). The contributions of behaviour change science towards dental public health practice: a new paradigm. Community Dent Oral Epidemiol, 43(1), 2-8. doi:10.1111/cdoe.12131

2. Azmoude, E., Aradmehr, M., \& Dehghani, F. (2018). Midwives' Attitude and Barriers of Evidence Based Practice in Maternity Care. Malaysian Journal of Medical Sciences, 25(3), 120-128. doi:10.21315/mjms2018.25.3.12

3. Bayes, S., Fenwick, J., \& Jennings, D. (2016). Readiness for Practice change: Evaluation of a tool for the Australian midwifery context. Women and Birth, 29, 240-244.

4. Bayes, S., Juggins, E., Whitehead, L., \& De Leo, A. (2019). Australian midwives' experiences of implementing practice change. Midwifery, 70,38-45. doi:https://doi.org/10.1016/j.midw.2018.12.012 
5. Birken, S. A., Powell, B. J., Presseau, J., Kirk, M. A., Lorencatto, F., Gould, N. J., . . Damschroder, L. J. (2017). Combined use of the Consolidated Framework for Implementation Research (CFIR) and the Theoretical Domains Framework (TDF): a systematic review. Implement Sci, 12(1), 2. doi:10.1186/s13012-016-0534-z

6. Braun, V., \& Clarke, V. (2006). Using thematic analysis in psychology. Qualitative REsearch in Psychology, 3(2), 77-101.

7. Cane, J., O'Connor, D., \& Michie, S. (2012). Validation of the theoretical domains framework for use in behaviour change and implementation research. Implement Sci, 7(37), 37. doi:10.1186/1748-5908-737

8. Davis, R., Campbell, R., Hildon, Z., Hobbs, L., \& Michie, S. (2015). Theories of behaviour and behaviour change across the social and behavioural sciences: a scoping review. Health Psychol Rev, 9(3), 323344. doi:10.1080/17437199.2014.941722

9. Fleming, A., Bradley, C., Cullinan, S., \& Byrne, S. (2014). Antibiotic prescribing in long-term care facilities: a qualitative, multidisciplinary investigation. BMJ Open, 4(11), e006442.

doi:10.1136/bmjopen-2014-006442

10. Friesen-Storms, J., Moser, A., van der Loo, S., Beurskens, A., \& Bours, G. (2015). Systematic implementation of evidence-based practice in a clinical nursing setting: a participatory action research project. J Clin Nurs, 24(1-2), 57-68. doi:10.1111/jocn.12697

11. Fuller, C., Besser, S., Savage, J., McAteer, J., Stone, S., \& Michie, S. (2014). Application of a theoretical framework for behavior change to hospital workers' real-time explanations for noncompliance with hand hygiene guidelines. Am J Infect Control, 42(2), 106-110. doi:10.1016/j.ajic.2013.07.019

12. Gallen, A., Kodate, N., \& Casey, D. (2019). How do nurses and midwives perceive their preparedness for quality improvement and patient safety in practice? A cross-sectional national study in Ireland. Nurse Educ Today, 76, 125-130. doi:10.1016/j.nedt.2019.01.025

13. Geerligs, L., Rankin, N. M., Shepherd, H. L., \& Butow, P. (2018). Hospital-based interventions: a systematic review of staff-reported barriers and facilitators to implementation processes. Implement Sci, 13(1), 36. doi:10.1186/s13012-018-0726-9

14. Glanz, K., \& Bishop, D. B. (2010). The role of behavioral science theory in development and implementation of public health interventions. Annu Rev Public Health, 31, 399-418. doi:10.1146/annurev.publhealth.012809.103604

15. Hanbury, A., \& Wood, H. (2018). Using behavioural science to explore patient perceptions. International Journal of Pharmaceutical and Healthcare Marketing, 12(4), 463-485. doi:10.1108/ijphm-04-2017-0020

16. Handley, M., Gorukanti, A., \& Cattamanchi, A. (2016). Strategies for implementing implementation science: a methodological overview. Emerg Med J, 33(9), 660-664. doi:10.1136/emermed-2015205461

17. Harvey, N., \& Holmes, C. (2012). Nominal group technique: an effective method for obtaining group consensus. Int J Nurs Pract, 18(2), 188-194. doi:10.1111/j.1440-172X.2012.02017.x 
18. Heydari, A., Mazlom, S., Ranjbar, H., \& Scurlock-Evans, L. (2014). A study of Iranian Nurses' and midwives' Knowledge, attitudes, and Implementation of Evidence-Based Practice: The time for change has arrived. Worldviews on Evidence Based Nursing, 11(5), 325-331.

19. Hogan, C., Barry, M., Burke, M., \& Joyce, P. (2011). Healthcare professionals' experiences of the implementation of integrated care pathways. Int J Health Care Qual Assur, 24(5), 334-347. doi:10.1108/09526861111139179

20. Kemmis, S., \& McTaggart, R. (1988). The action research planner (3rd edition ed.). Geelong: Deakin University Press.

21. Khammarnia, M., Haj Mohammadi, M., Amani, Z., Rezaeian, S., \& Setoodehzadeh, F. (2015). Barriers to implementation of evidence based practice in zahedan teaching hospitals, iran, 2014. Nurs Res Pract, 2015, 357140. doi:10.1155/2015/357140

22. Kislov, R., Waterman, H., Harvey, G., \& Boaden, R. (2014). Rethinking capacity building for knowledge mobilisation: developing multilevel capabilities in healthcare organisations. Implement Science, 9, 166. doi:10.1186/s13012-014-0166-0

23. Krefting, L. (1991). Rigor in qualitative research: the assessment of trustworthiness. Am J Occup Ther, 45(3), 214-222.

24. Langford, B. E., Schoenfeld, G., \& Izzo, G. (2002). Nominal grouping sessions vs focus groups. Qualitative Market Research: An International Journal, 5(1), 58-70. doi:10.1108/13522750210414517

25. Lynch, E. A., Luker, J. A., Cadilhac, D. A., Fryer, C. E., \& Hillier, S. L. (2017). A qualitative study using the Theoretical Domains Framework to investigate why patients were or were not assessed for rehabilitation after stroke. Clin Rehabil, 31(7), 966-977. doi:10.1177/0269215516658938

26. Mclntosh, M. J., \& Morse, J. M. (2015). Situating and Constructing Diversity in Semi-Structured Interviews. Glob Qual Nurs Res, 2, 2333393615597674. doi:10.1177/2333393615597674

27. Michie, S., Johnston, M., Abraham, C., Lawton, R., Parker, D., Walker, A., \& Psychological Theory, G. (2005). Making psychological theory useful for implementing evidence based practice: a consensus approach. Qual Saf Health Care, 14(1), 26-33. doi:10.1136/qshc.2004.011155

28. Michie, S., van Stralen, M. M., \& West, R. (2011). The behaviour change wheel: a new method for characterising and designing behaviour change interventions. Implement Sci, 6(42), 42. doi:10.1186/1748-5908-6-42

29. Moullin, J. C., Sabater-Hernandez, D., Fernandez-Llimos, F., \& Benrimoj, S. I. (2015). A systematic review of implementation frameworks of innovations in healthcare and resulting generic implementation framework. Health Res Policy Syst, 13(16), 16. doi:10.1186/s12961-015-0005-z

30. Nilsen, P., Neher, M., Ellstrom, P. E., \& Gardner, B. (2017). Implementation of Evidence-Based Practice From a Learning Perspective. Worldviews Evid Based Nurs, 14(3), 192-199. doi:10.1111/wvn.12212

31. Nithianandan, N., Gibson-Helm, M., McBride, J., Binny, A., Gray, K., East, C., \& Boyle, J. (2016). Factors affecting implementation of perinatal mental health screening in women of refugee background. Implement Sci, 11(1), 150. doi:10.1186/s13012-016-0515-2 
32. (2018). National compentency standards for the midwife. Nursing and Midwifery Board of Australia.

33. Pazandeh, F., Huss, R., Hirst, J., House, A., \& Baghban, A. (2015). An evaluation of the quality of care for women with low risk pregnanacy: The use of evidence-based practice during labour and childbirth in four public hospitals in Tehran. Midwifery, 31(11), 1045-1053. doi:10.1016/j.midw.2015.07.003

34. Powell, B., McMillen, J., Proctor, E., Carpenter, C., Griffey, R., Bunger, A., . . York, J. (2012). A compilation of strategies for implementing clinical innovations in health and mental health. Med Care Res Rev, 69(2), 123-157. doi:10.1177/1077558711430690

35. Powell, B. J., Waltz, T. J., Chinman, M. J., Damschroder, L. J., Smith, J. L., Matthieu, M. M., . . Kirchner, J. E. (2015). A refined compilation of implementation strategies: results from the Expert Recommendations for Implementing Change (ERIC) project. Implement Sci, 10, 21. doi:10.1186/s13012-015-0209-1

36. Schneider, Z., \& Whitehead, D. (2013). Nursing and Midwifery Research: Methods and appraisal for evidence-based practice 4th edition. Australia: Mosby Elsevier.

37. Tabak, R., Khoong, E., Chambers, D., \& Brownson, R. (2012). Bridging research and practice: models for dissemination and implementation research. Am J Prev Med, 43(3), 337-350.

doi:10.1016/j.amepre.2012.05.024

38. Tracy, S., Hartz, D., Nicholl, M., McCann, Y., \& Latta, D. (2005). An integrated service network in maternity-the implementation of a midwifery-led unit. Aust Health Rev, 29(3), 332-339. doi:10.1071/ah050332

39. Vogel, J., Moore, J., Timmings, C., Khan, S., Khan, D., Defar, A., . . Gulmezoglu, A. (2016). Barriers, Facilitators and Priorities for Implementation of WHO Maternal and Perinatal Health Guidelines in Four Lower-Income Countries: A GREAT Network Research Activity. PLoS One, 11(11), e0160020. doi:10.1371/journal.pone.0160020

\section{Supplementary Files}

This is a list of supplementary files associated with this preprint. Click to download.

- Declarations.docx

- Declarations.docx

- SRQRChecklist.IS.docx

- SRQRChecklist.IS.docx

- Contributionstothereserach.IS.docx

- Contributionstothereserach.IS.docx 